\title{
Investigation of the effect of quercetin in an experimental oxygen-induced retinopathy model
}

\author{
Abdulgani Kaymaz', Fatih Ulas', Sevilay Erimsah ${ }^{2}$, Cansu Kara Oztabag ${ }^{3}$ \\ ${ }^{1}$ Department of Ophthalmology, Bolu Abant Izzet Baysal University, Faculty of Medicine, Bolu, Turkey \\ ${ }^{2}$ Department of Histology and Embryology, Bolu Abant Izzet Baysal University, Faculty of Medicine, Bolu, Turkey \\ ${ }^{3}$ Department of Interdisciplinary Neuroscience, Bolu Abant Izzet Baysal University, Institute of Health Sciences, Bolu, \\ Turkey
}

\section{ABSTRACT}

\begin{abstract}
Aim: To investigate of the effect of intraperitoneal (IP) quercetin and bevacizumab on oxygen-induced retinopathy (OIR) model in rats.

Methods: In the study, 28 newborn rats were used. The OIR model was performed with the 50/10\% oxygen technique. The study consisted of four groups as a control group (Group I) and OIR groups (Group II, III, and IV). IP injection applied to all groups on the postnatal day (PND) 14. Groups I and II were performed $0.9 \%$ $\mathrm{NaCl}$, Group III was performed IP bevacizumab, and Group IV was performed IP quercetin. All animals were sacrificed on PND 18.
\end{abstract}

Results: Based on the data obtained from immunohistochemical and histopathological examinations, the number of vascular endothelial cell (VEC), vascular endothelial growth factor (VEGF), and tumor necrosis factor- $\alpha(\mathrm{TNF}-\alpha)$ levels were significantly reduced in Group III and IV compared to Group II. VECs levels were $0 \pm 0,32.69 \pm 5.77,2.92 \pm 0.63$, and 3.64 \pm 0.36 in Group I, Group II, Group III, and Group IV, respectively $(p<0.001)$. Likewise, VEGF values were $0.15 \pm 0.01,7.57 \pm 1.80,2.45 \pm 0.45$, and $2.46 \pm 0.49$, respectively $(p<0.001)$. As well as TNF- $\alpha$ values were $0.06 \pm 0.01,8.22 \pm 2.24,2.32 \pm 0.32$, and $2.29 \pm 0.26$ in Group I, Group II, Group III, and Group IV, respectively $(p<0.001)$. There was no significant difference between Group III and Group IV in terms of VEC, VEGF and TNF- $\alpha$ values (range of p values was 0.96-1.00).

Conclusion: The results of the present study showed that quercetin administration significantly reduced the VEC number and suppressed VEGF and TNF- $\alpha$. Quercetin's anti-inflammatory and anti-angiogenesis effect was found to be similar to bevacizumab.

Keywords: Oxygen-induced retinopathy, neovascularization, bevacizumab, quercetin, VEGF, TNF- $\alpha$, rats.

$\triangle$ Dr. Abdulgani Kaymaz.

Department of Ophthalmology, Bolu Abant Izzet Basal University, Faculty of Medicine, Bolu, Turkey

E-mail: kaymaz9@hotmail.com

Received: 2021-01-31 Revised: 2021-02-11

Accepted: 2021-02-20 / Published online: 2021-04-01

\section{Introduction}

Retinopathy of prematurity (ROP) can be mimicked in animals using the oxygen-induced retinopathy (OIR) model. With this model, the vaso-obliterative and neovascularization (NV) phases of ROP can be simulated with high reproducibility. Therefore, the treatment outcome of anti-angiogenic drugs can be assessed efficiently [1].

Diabetic retinopathy (DRP), ROP, and agerelated macular degeneration (AMD) are diseases that are seen as common in ophthalmology clinics that may result in vision loss and are associated with retinal angiogenesis and choroidal angiogenesis [2,3]. Pro- and anti-angiogenic factors are released in 
response to hypoxia that occurred in choroid and retina. There are many reasons that trigger the neovascularization (NV) in the eye, but one of the most well-known reasons is vascular endothelial growth factor (VEGF) [4]. Stimulation of VEGF receptor 2 (VEGFR2) via VEGF creates various intracellular signaling in vascular endothelial cells (VECs), including proliferation, migration, morphogenesis and increased permeability [5]. Likewise, another potent angiogenic molecule is tumor necrosis factor- $\alpha$ (TNF- $\alpha)$ that controls the genes encoding adhesion molecules and angiogenic mediators [6].

As a monoclonal antibody, bevacizumab inhibits VEGF, which is an angiogenic cytokine. It prevents pathological angiogenesis through promoting vascular leakage and growth [7]. It has been documented that off-label use of bevacizumab is effective in the treatment of many intraocular vascular proliferation retinal diseases associated with ischaemic retinopathy, such as DRP, ROP, and retinal vein occlusion (RVO) [8,9].

Quercetin (3,3', 4',5,7-penthydroxy flavone) belongs to the polyphenol family, which is one of the most abundant flavonoid in the diet, and is present in many fruits and vegetables, including onions, apples and grapes [10]. Previous in vivo and in vitro studies have shown that quercetin decreases oxidative stress, retinal neurodegeneration, and $\mathrm{NV}$ effectively [11,12]. Moreover, VEGF-induced cell proliferation, migration and tube formation was inhibited by quercetin [13]. Likewise, quercetin-treated retinas showed significantly lower levels of TNF- $\alpha$ and other proinflammatory cytokines [14].

The impact of quercetin on many organs, including the eye has been extensively studied. However, less is known about the impacts of intraperitoneal (IP) administration of quercetin on anti-VEGF factors using OIR models. The objective of the present study was therefore to investigate the effect of quercetin on VEGF, TNF- $\alpha$, and VEC using OIR models and comparing the results with those of bevacizumab.

\section{Materials and methods}

This study was conducted in compliance with the recommendations of the ARVO Statement for Animal Use in Ophthalmic and Vision Studies. The research was also accepted by the Bolu Abant Izzet Baysal University Experimental Animal Studies Ethics Committee (Date / decision no: 2018/35).

\section{Creating the OIR Model}

In the present study, 28 newborn Sprague Dawley rats were used. All animals were kept in a controlled room with $22-24{ }^{\circ} \mathrm{C}$ temperature and $45-60 \%$ relative humidity with $12 \mathrm{~h}$ light: $12 \mathrm{~h}$ dark conditions. The food and water were provided ad libitum. In the 50/10 OIR model, lactating pups were placed in an oxygen regulated environment with their mothers within $4 \mathrm{~h}$ following birth, where they were exposed to $50 \%$ oxygen for $24 \mathrm{~h}$ followed by $10 \%$ oxygen for $24 \mathrm{~h}$ [15]. This cycle was repeated until postnatal day (PND) 14 seven times. Daily monitoring of oxygen levels was performed and was calibrated as needed. Likewise, daily carbon dioxide levels in the cage were also monitored and sufficient gasflow was maintained through flushing it from the system and adding soda lime. Pups were put into a room with ambient air for 4 days on PND 14.

The experiment was performed with four groups with seven animals per group. The injections were administered intraperitoneally to all animals only once on day 14 of the creation of the OIR model. 
Group I: The healthy group administered 0.01 $\mathrm{ml}$ ip $0.9 \% \mathrm{NaCl}$ solution (control group). Group II: OIR group administered $0.01 \mathrm{ml}$ ip $0.9 \% \mathrm{NaCl}$ solution (Untreated OIR group). Group III: OIR group treated with $0.01 \mathrm{ml}$ ip bevacizumab (2.5 $\mathrm{mg} / \mathrm{kg}$ body weight) (Altuzan, Roche, Istanbul, Turkey). Group IV: OIR group treated with $0.01 \mathrm{ml}$ ip quercetin (20 $\mathrm{mg} / \mathrm{kg}$ body weight) (Sigma-Aldrich, St.Louis, USA) [16]. It is assumed that the OIR pattern is best formed PNDs 18-20. Therefore, on PND 18, all animals were sacrificed with high dose intracardiac anesthesia, and the right eyes were enucleated [17]. The tissues were stored for further histopathological and immunohistochemical analyses.

\section{Haematoxylin and eosin $(H \& E)$ staining}

The eyes were set in 10 percent neutral buffered formalin in PBS overnight at $4{ }^{\circ} \mathrm{C}$ and then embedded in paraffin. The serial sections $(4 \mu \mathrm{m}$ thick) of the whole eye were performed sagittal that was parallel to the optic nerve and stained with H\&E. The nuclei of retinal VECs on the vitreal side of the retinal inner limiting membrane (ILM) were counted by an objective observer blind to treatment in ten parts for each eye at 400x magnification and the mean number of nuclei of endothelial cells for each eye was determined for each community [18].

\section{Immunohistochemistry staining}

A biotin-streptavidin HRP detection kit (ab93697; Abcam, Cambridge, UK) was used to perform immunohistochemistry. Antigen retrieval was performed with citrate buffer. $3 \%$ $\mathrm{H}_{2} \mathrm{O}_{2}$ in methanol was used for blocking endogenous peroxidases for $15 \mathrm{~min}$. In order to eliminate non-specific binding, a blocking serum was used for the pretreatment of the sections. Then, the sections were incubated overnight with the following antibodies: anti-
TNF alpha polyclonal antibody (1:250 dilution; ab183896; Abcam) and anti-VEGF monoclonal antibody (1:50 dilution; sc-7269; Santa, Santa Cruz, CA, USA) at $4^{\circ} \mathrm{C}$. An appropriate nonimmune immunoglobulin $\mathrm{G}$ was used as a primary antibody replacement to perform negative control incubations. Then, biotinylated secondary antibodies were used to incubate the tissues. The peroxide complex was visualized using 3,3-diaminobenzidine. Finally, Mayer's hematoxylin (Invitrogen, California, USA) was used to counterstain the tissues, and then they were mounted with Entellan (Merck, Darmstadt, Germany) on glass slides. The images were observed under a light microscope (Leica DM 1000, Germany) and photos were taken using Leica DMC 2900 (CH-9435 Heerbrugg, Germany).

The following scales were used to grade of section: no expression (0), mild (1), moderate (2), strong (3), and very strong expression (4) by two independent observers blind to treatment. The percentage of positive cells was described as (0), < 5\%; (1), $6 \%$ to $15 \%$; (2), $16 \%$ to $50 \%$; (3), $51 \%$ to $80 \%$; and (4), > $80 \%$ of positive cells [19]. The mean value of the three retinal sections of each rat was determined.

\section{Statistical analyses}

In the present study, the data were analyzed using SPSS statistical software package, version 25.0 (SPSS Inc., Chicago, IL, USA). The data were reported as mean \pm standard deviations (SDs) for each data set. A statistical significance was considered if $p<0.05$. The homogeneity and normality of the sample distribution of data were determined using Levene's test and Kolmolgorov Smirnov test, respectively. The statistical analyses of the data was performed with one-way analysis of variance test and post hoc Tukey's test. 


\section{Results}

Representative hematoxylin and eosin-stained retinas of all groups are presented in Figure 1. In the untreated OIR group (Group II), abundant longitudinal and transverse aberrant microvessels were noted in ILM. The nuclei of VECs were counted to quantify NV. Bevacizumab (Group III) and Quercetin (Group IV) administered groups showed a reduced number of VECs breaching ILM as well as improvement of vascular tufts and dilated vessels. In Group I (control group), there were no VEC nuclei detected on the vitreal side of the ILM of the retina (Figure 1).
However, the retinal VEC nucleus number was significantly higher in Group II compared with Group III and Group IV (Figure 1). On the other hand, VEC nucleus number were similar between Group III and Group IV ( $p=0.960$; Figure 1).

VEGF and TNF- $\alpha$ levels in the control and OIR groups were determined through immunohistochemistry (Table 1). VEGFpositive staining was apparent in the retinas of OIR rats, especially in the GCL and INL layers (Figure 2). The intensity of the VEGF staining was reduced in Group III and Group IV. Semi-

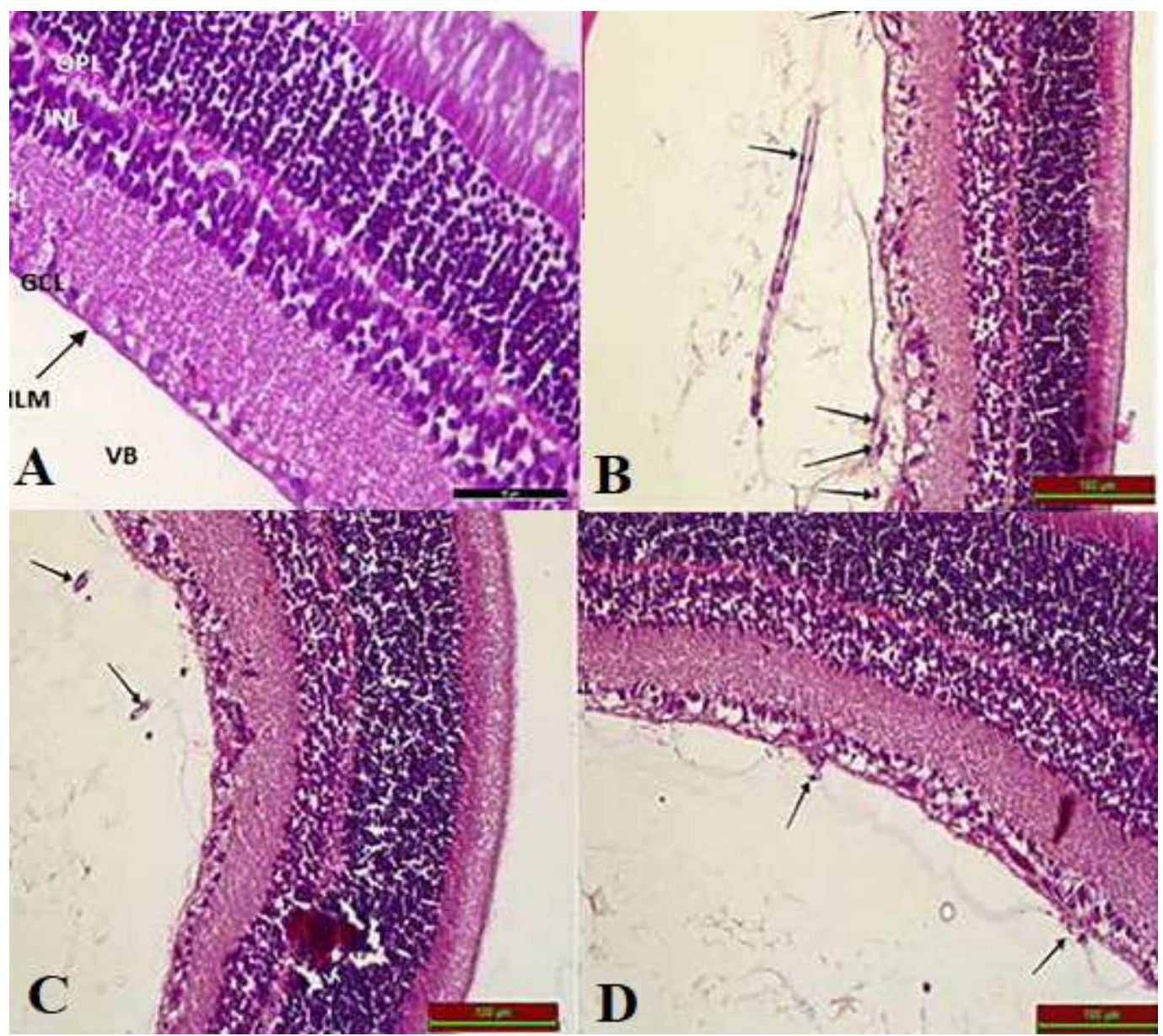

Figure 1. Haematoxylin and Eosin staining of neovascularization in retinal tissues. A: Control group (Group I). B: Untreated OIR group (Group II). C: OIR group treated with intraperitoneal bevacizumab (Group III). D: OIR group treated with intraperitoneal quercetin (Group IV). Quantitative analysis was performed by counting the nuclei of retinal vascular endothelial cells (arrows) on the vitreal side of the inner limiting membrane in 10 sections from each eye. GCL: Ganglion cell layer, ILM: Inner limiting membrane, INL: Inner nuclear layer, IPL: Inner plexiform layer, OLM: Outer limiting membrane, ONL: Outer nuclear layer, OPL: Outer plexiform layer, PL: Photoreceptor layer, VB: Vitreous body. 
Table 1. The results of H\&E and immunohistochemical staining in the OIR model.

\begin{tabular}{|l|l|l|l|l|l|}
\hline Variables & Group I & Group II & Group III & Group IV & $\boldsymbol{P}^{*}$ \\
\hline VEC nuclei & 0 & $32.69 \pm 5.77$ & $2.92 \pm 0.63$ & $3.64 \pm 0,36$ & $<0.001$ \\
\hline TNF- $\boldsymbol{\alpha}$ & $0.06 \pm 0.01$ & $8.22 \pm 2.24$ & $2.32 \pm 0.32$ & $2.29 \pm 0,26$ & $<0.001$ \\
\hline VEGF & $0.15 \pm 0.01$ & $7.57 \pm 1.80$ & $2.45 \pm 0.45$ & $2.46 \pm 0,49$ & $<0.001$ \\
\hline
\end{tabular}

Values: mean \pm SD. H\&E: Hematoxylin \& eosin. OIR: oxygen-induced retinopathy.

*One-way analysis of variance test

Group I: $0.01 \mathrm{ml}$ intraperitoneal saline solution $(0.9 \% \mathrm{NaCl})$ was administered without creating an OIR model (control group).

Group II: $0.01 \mathrm{ml}$ intraperitoneal saline solution was administered following the creation of the OIR model (Untreated OIR group).

Group III: $0.01 \mathrm{ml}$ intraperitoneal bevacizumab $(2.5 \mathrm{mg} / \mathrm{kg}$ body weight) (Altuzan, Roche, Istanbul, Turkey) was administered in the OIR group.

Group IV: $0.01 \mathrm{ml}$ intraperitoneal quercetin (20 mg/kg body weight) (Sigma-Aldrich, St.Louis, USA) was administered in the OIR group.

TNF- $\alpha$ : Tumor necrosis factor- $\alpha, V E C$ : Vascular endothelial cell, VEGF: Vascular endothelial growth factor.

quantification of VEGF immune-intensity demonstrated that VEGF staining was significantly decreased in Group III and Group IV compared with Group II ( $p<0.001$ per group compared). Moreover, quercetin treatment reversed the oxygen-induced VEGF elevation that was compatible with bevacizumab.

Semi-quantification of TNF- $\alpha$ immuneintensity demonstrated that TNF- $\alpha$ staining was significantly decreased in Group III and Group IV compared with Group II ( $p<0.001$ per group compared). Quercetin treatment reversed the oxygen-induced TNF- $\alpha$ elevation (Figure 3 ).

Retinal VEGF and TNF- $\alpha$ expression were significantly increased in Group II compared to Group I, III, and IV (Table 1 and Figure 2). On the other hand, levels of VECs, VEGF, and TNF- $\alpha$ were significantly decreased in Group III and Group IV compared to Group II $(p<0.001$; Table 1).
Post hoc test results of VECs, VEGF and TNF$\alpha$ in Group II were significantly higher compared to Group I, III, and IV ( $p<0.001$ per group compared). While the nucleus number of VECs was similar between Group I and III, VEGF and TNF- $\alpha$ levels were found to be significantly reduced in Group III $(p=0.30$, $p=0.001$, and $p=0.008$, respectively). Likewise, the nucleus number of VEC was similar between Group I and IV ( $p=0.14)$. In addition, VEGF and TNF levels were significantly reduced in Group IV $(p=0.001$ and $p=0.009$, respectively). The nucleus number of VEC, as well as VEGF and TNF levels, were similar between Group III and IV $(p=0.96, p=1.000$, and $p=1.000$, respectively).

\section{Discussion}

Retinal NVs are the primary reasons for vision loss in patients, including neovascular AMD, 


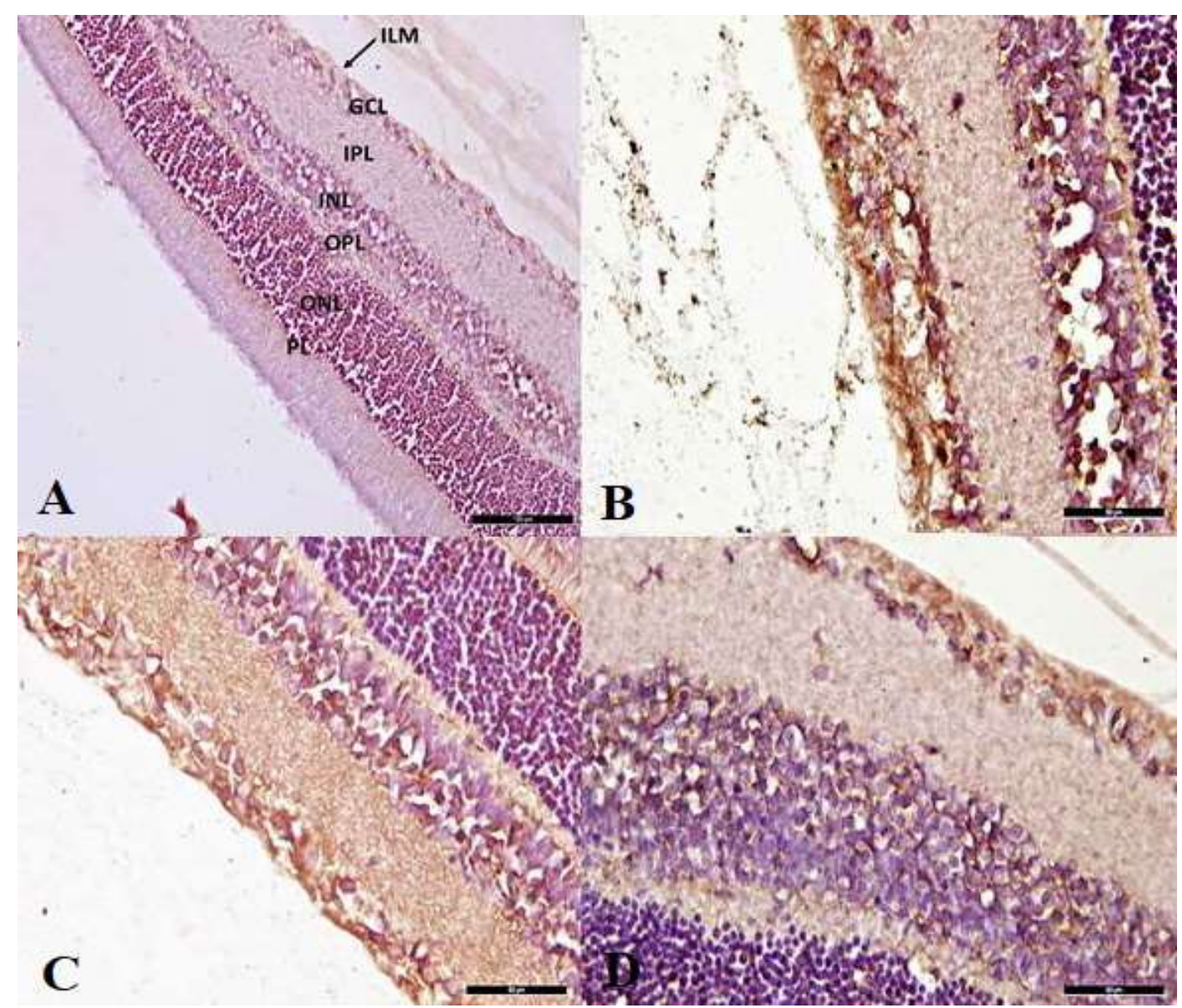

Figure 2. VEGF expressions were determined by immunohistochemistry in non-treated OIR, and OIR rats treated with quercetin or bevacizumab. A: Control group (Group I). B: Untreated OIR group (Group II). C: OIR group treated with intraperitoneal bevacizumab (Group III). D: OIR group treated with intraperitoneal quercetin (Group IV). GCL: Ganglion cell layer, ILM: Inner limiting membrane, INL: Inner nuclear layer, IPL: Inner plexiform layer, ONL: Outer nuclear layer, OPL: Outer plexiform layer, PL: Photoreceptor layer.

proliferative DRP, RVO, and ROP. The results of the present study demonstrated that ip administration of quercetin following an OIR model histopathologically suppressed the retinal NVs and inflammatory cell infiltration as well as significantly reduced the number of VECs, VEGF, and TNF- $\alpha$ levels compared to the non-treated OIR groups. The data obtained with quercetin administration were comparable to that of the bevacizumab administration.

Stimulation of VECs in the case of retinal hypoxia could increase the mRNA transcript abundance of various genes, including growth factor associated genes, such as VEGF and
TNF- $\alpha$ [20]. Chen et. al. suggested that various stages of angiogenesis, including proliferation, migration, and tube formation of choroidal and retinal VECs, were inhibited following quercetin administration in vitro [21]. Likewise, $\mathrm{Li}$ et al. found that VEGF-induced choroidal and retinal angiogenesis were inhibited by in vitro quercetin administration [13]. It was reported that quercetin could inhibit blood vessel development and reduce cell viability, cell proliferation, and tube formation [22]. Similar to the previously published studies, our results suggested that quercetin significantly reduced VECs in the experimental 


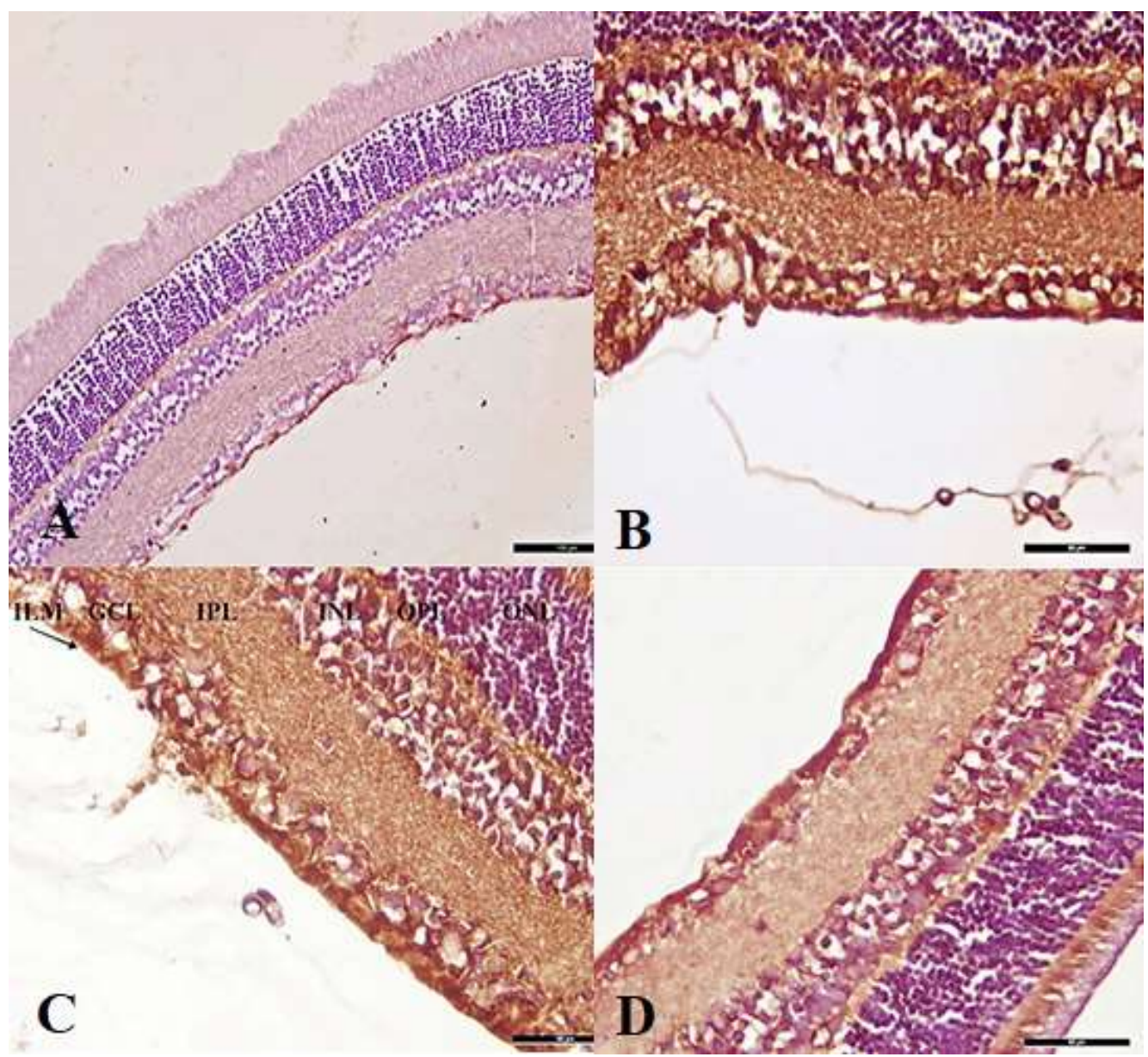

Figure 3. TNF- $\alpha$ expressions were determined by immunohistochemistry in non-treated OIR, and OIR rats treated with quercetin or bevacizumab. A: Control group (Group I). B: Untreated OIR group (Group II). C: OIR group treated with intraperitoneal bevacizumab (Group III). D: OIR group treated with intraperitoneal quercetin (Group IV). GCL: Ganglion cell layer, ILM: Inner limiting membrane, INL: Inner nuclear layer, IPL: Inner plexiform layer, ONL: Outer nuclear layer, OPL: Outer plexiform layer.

OIR model, and suppressed the synthesis of VEGF and TNF- $\alpha$.

Currently, intravitreal bevacizumab has been successfully used for the treatment of DRP, ROP, RVO, and AMD. Many studies have reported that it has strong anti-VEGF properties and similar efficiency compared to the other intravitreal agents (such as aflibercept and ranibizumab) [7,8]. Significant improvement was shown in the zone I ROP following treatment with intravitreal bevacizumab as an anti-VEGF monotherapy and was comparable to the conventional laser therapy [23]. In the current study, the administration of quercetin and bevacizumab showed strong anti-VEGF properties compared to the non-treated OTNF$\alpha$ is an important mediator of retinal neuroinflammation and neurodegeneration. It has previously demonstrated that the cytokines and chemokines were strongly suppressed by bevacizumab in the experimental OIR model [24]. Likewise, cytokine levels were significantly reduced in quercetin-treated retinas compared to the proliferative diabetic retinas [25]. To the best of our knowledge, there are no previous studies examining the impact of quercetin administration on TNF- $\alpha$ levels using experimental OIR models. The results of the current study were comparable to that of DRP models. Similar to the bevacizumab 
administration, quercetin treatment in the OIR model significantly suppressed TNF- $\alpha$ compared to the non-treated OIR group.

The single-dose i.p. administration of quercetin in the OIR model and examination of only VECs, VEGF and TNF- $\alpha$ limited the strength of the study. In addition, dose-response relationship, pharmacokinetic and functional analyses were not evaluated in the current study. Therefore, future studies are required to determine the optimal dosing protocol, especially for intravitreal application. Although the short-term effects were quite promising, future studies should be performed to confirm the long-term effects. Despite all these limitations, it was found in the present study that quercetin has comparable efficacy to bevacizumab, the anti-VEGF agent currently used in routine therapy.

In conclusion, the results of the present study demonstrated that quercetin administration as a therapeutic agent may ameliorate the severity of OIR in a rat model. Moreover, the effects obtained by quercetin treatment using the OIR model were comparable to the effects obtained following bevacizumab administration. Therefore, quercetin administration is promising for the treatment of diseases causing vision loss in patients, including ROP, DRP, RVO, and AMD. However, more studies are required in the future for a better conclusion.

\section{Acknowledgement}

The authors thank Mr. Ibrahim Ethem Torun for assistance with digital imaging.

Funding: Supported by the Scientific Research Project Coordination Unit of Bolu Abant Izzet Baysal University.

Conflict of Interest: The authors declare that they have no conflict of interest.

Ethical statement: The study was approved by the Ethics committee of Bolu Abant Izzet Baysal
University Experimental Animal Studies (Date / decision no: 2018/35).

\section{Open Access Statement}

This is an open access journal which means that all content is freely available without charge to the user or his/her institution under the terms of the Creative Commons Attribution NonCommercial License

(http://creativecommons.org/licenses/bync/4.0). Users are allowed to read, download, copy, distribute, print, search, or link to the full texts of the articles, without asking prior permission from the publisher or the author.

\section{References}

[1]Park SW, Kim JH, Kim KE, et al. Betalapachone inhibits pathological retinal neovascularization in oxygen-induced retinopathy via regulation of HIF-1alpha. J Cell Mol Med. 2014;18(5):875-84.

[2]Ferris FL, Fine SL, Hyman L. Age-related macular degeneration and blindness due to neovascular maculopathy. Arch Ophthalmol. 1984;102(11):1640-42.

[3]Engerman RL. Pathogenesis of diabetic retinopathy. Diabetes. 1989;38(10):12031206.

[4]Sato T, Kusaka S, Shimojo H, et al. Vitreous levels of erythropoietin and vascular endothelial growth factor in eyes with retinopathy of prematurity. Ophthalmology. 2009;116(9):1599-1603.

[5]Seo S, Suh W. Antiangiogenic effect of dasatinib in murine models of oxygeninduced retinopathy and laser-induced choroidal neovascularization. Mol Vis. 2017;23:823-31.

[6]Grilli M, Chiu JJ, Lenardo MJ. NF-kappa B and Rel: participants in a multiform transcriptional regulatory system. Int Rev Cytol. 1993;143:1-62. 
[7]Moja L, Lucenteforte E, Kwag KH, et al. Systemic safety of bevacizumab versus ranibizumab for neovascular age-related macular degeneration. Cochrane Database Syst Rev. 2014(9):CD011230.

[8]Arevalo JF, Sanchez JG, Fromow-Guerra J, et al. Comparison of two doses of primary intravitreal bevacizumab (Avastin) for diffuse diabetic macular edema: results from the Pan-American Collaborative Retina Study Group (PACORES) at 12-month follow-up. Graefe's archive for clinical and experimental ophthalmology = Albrecht von Graefes Archiv fur klinische und experimentelle Ophthalmologie. 2009;247(6):735-43.

[9]Mitchell P, Smith W, Wang JJ, et al. Prevalence of diabetic retinopathy in an older community. The Blue Mountains Eye Study. Ophthalmology. 1998;105(3):40611.

[10]Lamson DW, Brignall MS. Antioxidants and cancer, part 3: quercetin. Altern Med Rev. 2000;5(3):196-208.

[11]Chen B, He T, Xing Y, et al. Effects of quercetin on the expression of MCP-1, MMP-9 and VEGF in rats with diabetic retinopathy. Exp Ther Med. 2017;14(6):6022-26.

[12] Ola MS, Ahmed MM, Shams S, et al. Neuroprotective effects of quercetin in diabetic rat retina. Saudi J Biol Sci. 2017;24(6):1186-94.

[13] Li F, Bai Y, Zhao M, et al. Quercetin inhibits vascular endothelial growth factor-induced choroidal and retinal angiogenesis in vitro. Ophthalmic Res. 2015;53(3):109-16.

[14] Kumar B, Gupta SK, Nag TC, et al. Retinal neuroprotective effects of quercetin in streptozotocin-induced diabetic rats. Exp Eye Res. 2014;125:193-202.
[15]Penn JS, Henry MM, Tolman BL. Exposure to alternating hypoxia and hyperoxia causes severe proliferative retinopathy in the newborn rat. Pediatr Res. 1994;36(6):72431.

[16] Arikan S, Ersan I, Karaca T, et al. Quercetin protects the retina by reducing apoptosis due to ischemia-reperfusion injury in a rat model. Arq Bras Oftalmol. 2015;78(2):100104.

[17] Hartnett ME. The effects of oxygen stresses on the development of features of severe retinopathy of prematurity: knowledge from the 50/10 OIR model. Doc Ophthalmol. 2010;120(1):25-39.

[18]Park K, Chen Y, Hu Y, et al. Nanoparticlemediated expression of an angiogenic inhibitor ameliorates ischemia-induced retinal neovascularization and diabetesinduced retinal vascular leakage. Diabetes. 2009;58(8):1902-13.

[19] Gocmez SS, Yazir Y, Sahin D, et al. The effect of a selective neuronal nitric oxide synthase inhibitor 3-bromo 7-nitroindazole on spatial learning and memory in rats. Pharmacol Biochem Behav. 2015;131:1925.

[20]Fraisl P, Mazzone M, Schmidt T, et al. Regulation of angiogenesis by oxygen and metabolism. Dev Cell. 2009;16(2):167-79.

[21]Chen Y, Li XX, Xing NZ, et al. Quercetin inhibits choroidal and retinal angiogenesis in vitro. Graefe's archive for clinical and experimental ophthalmology = Albrecht von Graefes Archiv fur klinische und experimentelle Ophthalmologie. 2008;246(3):373-78.

[22]Zhao D, Qin C, Fan X, et al. Inhibitory effects of quercetin on angiogenesis in larval zebrafish and human umbilical vein endothelial cells. Eur J Pharmacol. 2014;723:360-67. 
[23] Mintz-Hittner HA, Kennedy KA, Chuang $\mathrm{AZ}$, et al. Efficacy of intravitreal bevacizumab for stage $3+$ retinopathy of prematurity. N Engl J Med. 2011;364(7):603-15.

[24]Lafreniere JD, Toguri JT, Gupta RR, et al. Effects of intravitreal bevacizumab in Grampositive and Gram-negative models of ocular inflammation. Clin Exp Ophthalmol. 2019;47(5):638-45.

[25] Kumar B, Gupta SK, Nag TC, et al. Retinal neuroprotective effects of quercetin in streptozotocin-induced diabetic rats. Exp Eye Res. 2014;125:193-202. 